\title{
Untersuchung und Berechnung der Lebensdauer raumgängiger Kunststoff-Gleitketten
}

\author{
Dr.-Ing. P. Auerbach \\ Prof. Dr.-Ing. K. Nendel \\ TU Chemnitz, Professur FördertechniK
}

Der Artikel beschreibt Versuche zur Bestimmung der Dauerfestigkeit von Kunststoff-Gleitketten auf Prüfmaschine und Testförderstrecken. Es werden die ermittelten Kennwerte dargestellt und ein Ansatz zur rechnerischen Abschätzung der Lebensdauer vorgeschlagen. Abschließend wird ein Computerprogramm zur einfachen Dimensionierung von Gleitketten-Fördersystemen auf Grundlage der Ergebnisse vorgestellt.

\section{Einleitung}

Fördersysteme mit Kunststoff-Gleitketten zum Transport leichter Stückgüter unterliegen vielfältigen Beanspruchungen, die in einem bereits veröffentlichten Artikel [Auerbach06] mit dem Titel „Untersuchung der Beanspruchung und des Zugkraftverlaufs raumgängiger Gleitketten“ dargestellt wurden. Im Ergebnis der dort beschriebenen Untersuchungen wurde die zeitabhängige Kettenzugkraft als Hauptbeanspruchung identifiziert und es konnten genauere Gleichungen zur Berechnung der Zugkräfte für Kurven mit Gleitreibung aufgestellt werden.

Bei der Dimensionierung von Gleitketten-Fördersystemen wird geprüft, ob die berechnete Kettenzugkraft kleiner als die vom Hersteller angegebene zulässige Zugkraft ist. Eine genaue Auslegung ist nur möglich, wenn sowohl die wirkende als auch die zulässige Zugkraft zutreffend sind. Bislang fehlt jedoch eine Normung bzw. Definition der zulässigen Kettenzugkraft, so dass jeder Hersteller diesen Wert anders ermittelt. Die Angaben verschiedener Hersteller können daher nicht direkt miteinander verglichen werden und es resultieren unterschiedliche Lebensdauern der Ketten, die bislang aber nicht rechnerisch abgeschätzt werden können.

Voraussetzung für eine verbesserte Auslegung ist eine genauere Untersuchung der Festigkeitseigenschaften seitenbeweglicher Kunststoff-Gleitketten unter dynamischer Beanspruchung. Dabei muss auch dem vermuteten Einfluss der Streckenführung auf die Lebensdauer [Auerbach06] Rechnung getragen werden, der bislang nicht untersucht worden ist. Der Artikel beschreibt die Ergebnisse derartiger Untersuchungen an seitenbeweglichen Fingerketten mit $65 \mathrm{~mm}$ Breite aus drei verschiedenen Kunststoffen. Weiterhin wird ein Ansatz vorgeschlagen, der erstmals eine rechnerische Abschätzung der Lebensdauer ermöglicht. Der Artikel schließt mit der Beschreibung einer Berechnungssoftware, die entwickelt wurde, um die Dimensionierung von Gleitketten-Fördersystemen auf der Grundlage des neuen Ansatzes zu erleichtern.

\section{Festigkeitsuntersuchungen}

\subsection{Prüfmethoden}

Bei der Untersuchung der Dauerfestigkeiten von Gleitketten bestehen zwei konkurrierende Forderungen, die gegeneinander abgewogen werden müssen:

1. Prüfung unter möglichst realitätsnahen Beanspruchungsbedingungen, um Werte zu erhalten, die auf reale Förderstrecken zutreffen,

2. Abkürzung der generell sehr langen Versuchszeiten durch weitgehende Vereinfachung von Belastungsfall und Zeitverlauf.

Fasst man die realen Beanspruchungen von Gleitketten vereinfachend als einachsige, schwellende Zugbeanspruchung auf, kann eine dynamische Prüfmaschine zum Testen verwendet werden. Diese 
erlaubt relativ hohe Belastungsfrequenzen, die v.a. durch die innere Dämpfung und Erwärmung der Kunststoffe begrenzt werden, und folglich vergleichsweise kurze Versuchszeiten.

Der Einfluss der zusätzlichen Beanspruchungen v.a. in engen horizontalen Kurven auf die Lebensdauer kann mit der Prüfmaschine nicht untersucht werden. Um unter diesen Belastungsverhältnissen prüfen zu können, wurden entsprechende Testförderstrecken verwendet. Aufgrund der erforderlichen Kettenlänge und der begrenzten Geschwindigkeit dauert ein Kettenumlauf (d. h. Lastwechsel) jedoch relativ lange, so dass diese Art der Prüfung sehr zeitaufwendig ist.

Es wurden mit beiden Anordnungen Versuche durchgeführt, um festzustellen, inwieweit die Ergebnisse übereinstimmen und ob durch eine günstige Kombination beider Verfahren verlässliche Werte mit verringertem Zeitaufwand erzielt werden können.

\subsection{Untersuchungen mit dynamischer Prüfmaschine}

Wie in der DIN 8153 T1 für Stahlketten empfohlen, wurden jeweils fünf Kettenglieder beidseitig kardanisch in der Prüfmaschine aufgehängt, um Biegebeanspruchungen zu vermeiden (Abbildung 1).

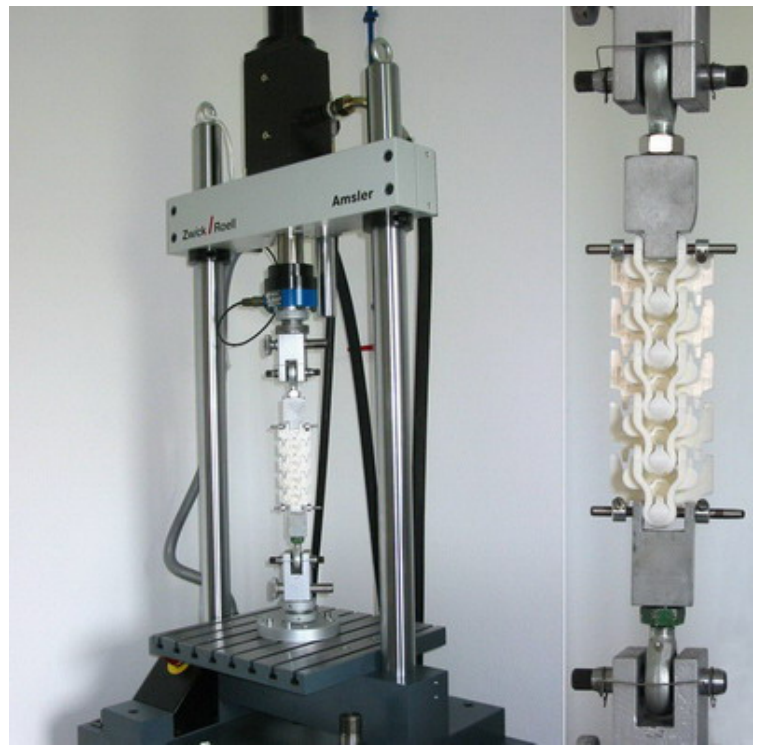

Abb. 1: Dynamische Prüfmaschine und Einspannvorrichtung für Kettenmuster

Geprüft wurden seitenbewegliche Fingerketten $\left(r_{\min }=150 \mathrm{~mm}\right)$ mit $65 \mathrm{~mm}$ Breite aus folgenden drei Werkstofftypen:

1. Delrin ${ }^{\circledR} 500 \mathrm{Al}$ (POM-Homopolymer),

2. Hostaform ${ }^{\circledR}$ LW90EWX (POM-Copolymer),

3. Celanex ${ }^{\circledR} 2002-2$ (PBT).

Dazu wurde zunächst für jeden Werkstoff die Bruchlast ermittelt und prozentual dazu (30, 40 und 50\%) die Amplituden der schwellenden Last für die Dauerversuche festgelegt. Die Kettenproben aus den genannten Werkstoffen wurden bei jeder Laststufe dreimal geprüft, um die Streubreite der Werte einschätzen zu können. Die sehr lange dauernden Prüfungen mit 30\% der Bruchlast konnten aufgrund begrenzter Prüfkapazitäten nur zwei Mal durchgeführt werden und wurden bei $10^{6}$ Lastwechseln abgebrochen. Die gewählte Belastungsfrequenz von $2 \mathrm{~Hz}$ gilt als guter Kompromiss [Crawford98] zwischen akzeptabler Versuchsdauer und der Vermeidung thermischen Versagens der Probe. Abbildung 2 zeigt die Ergebnisse in Form eines Wöhlerdiagramms mit logarithmischer Auftragung der mittleren Bruchspielzahlen bei einer bestimmten Amplitude der Prüflast.

Die im Diagramm gestrichelt dargestellten Verläufe in Richtung höherer Schwingspielzahlen basieren auf einer linearen Extrapolation des vorherigen Verlaufs. Dabei wird unterstellt, dass keine ausgeprägte 


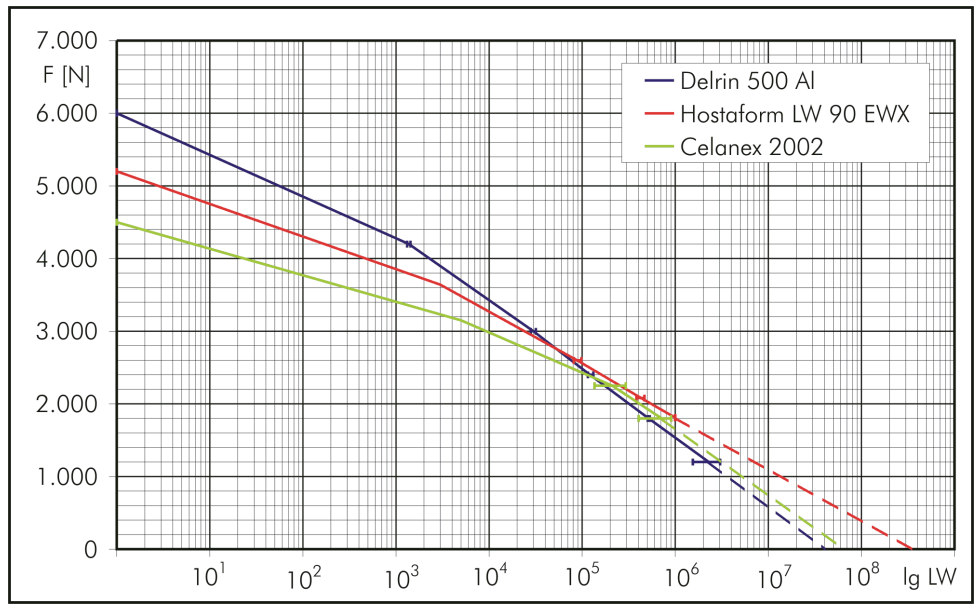

Abb. 2: Wöhlerlinien der untersuchten Werkstoffe

Dauerfestigkeit existiert, was bei vielen Kunststoffen auch gegeben ist [Hellerich04, S. 343]. Der Vergleich der drei Werkstoffe verdeutlicht, dass die statische Bruchkraft keine zuverlässige Beurteilung des Festigkeitsverhaltens unter dynamischer Belastung erlaubt. So hat der Werkstoff Delrin ${ }^{\circledR}$ die mit Abstand höchste statische Bruchkraft, schneidet aber bei der Langzeitfestigkeit schlechter ab. Die beiden anderen Werkstoffe zeigen einen geringeren Festigkeitsabfall und erzielen daher trotz geringerer statischer Bruchkraft eine bessere dynamische Festigkeit. Celanex ${ }^{\circledR}(\mathrm{PBT})$ weist mit im Mittel 30\% eine stärkere Streuung der Bruchspielzahlen auf als die beiden Polyacetal-Werkstoffe mit etwa $10 \%$.

\subsection{Untersuchungen auf Testförderstrecken}

Zum Vergleich mit den Versuchen auf der dynamischen Prüfmaschine wurde die Haltbarkeit der Ketten auf Testförderstrecken untersucht. Diese bestanden aus Traggestell, Antriebs- und Umlenkeinheit sowie verschiedenen Elementen zur Streckenführung (Geraden, Bogenräder, Gleitbögen), um deren Einfluss auf die Lebensdauer untersuchen zu können (Abbildung 3).

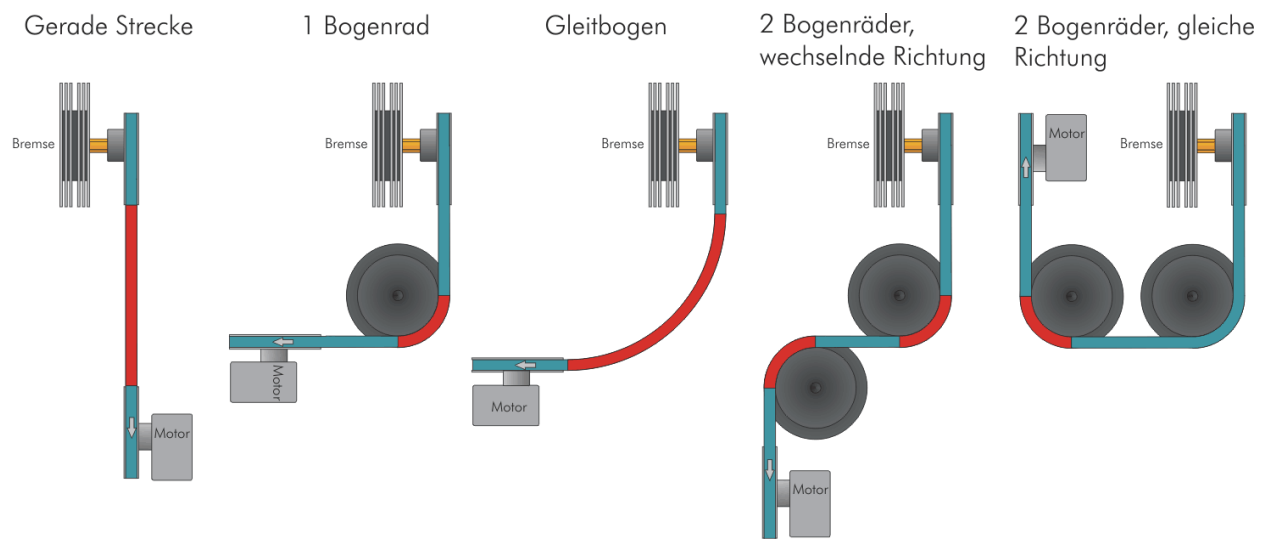

Abb. 3: Untersuchte Streckenführungen der Versuchsförderer

Die Bogenräder besaßen einen Nenndurchmesser von 300 mm, der Gleitbogen einen mittleren Radius von $700 \mathrm{~mm}$ und die Länge der geraden Förderstrecke betrug $1.000 \mathrm{~mm}$. Die Kettenbelastung wurde durch Abbremsen (Vorspannen) der Kette mittels einer an der Umlenkung angekuppelten Magnetpulverbremse realisiert. Durch Messungen der Kettenzugkraft bei verschiedenen Bremsmomenten bzw. angelegten Spannungen wurde der entsprechende Proportionalitätsfaktor ermittelt. Damit konnte später die gewünschte Zugkraft durch Einstellen einer bestimmten Spannung erzeugt werden. Die 
Bremskraft wurde mittels einer Momentenstütze während der gesamten Versuchsdauer regelmässig überwacht. Abbildung 4 zeigt den Aufbau einer derartigen Versuchsförderstrecke.

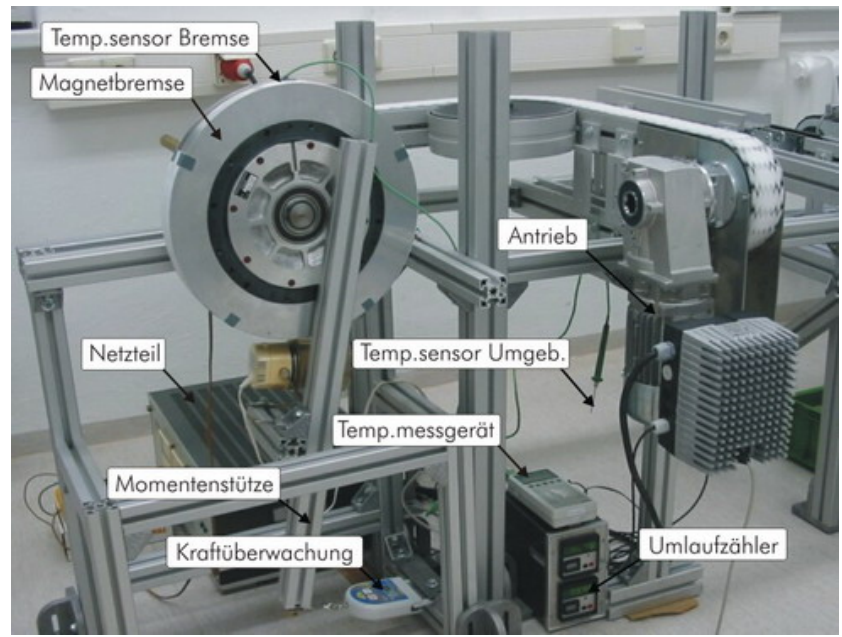

Abb. 4: Aufbau einer Versuchsförderstrecke

Die gezeigten Strecken wurden mit einer maximalen Kettenzugkraft von $1.000 \mathrm{~N}$ geprüft. Die Strecke mit einem Bogenrad wurde zusätzlich bei 600 und $850 \mathrm{~N}$ geprüft, um den Festigkeitsabfall der Kette in Form einer Wöhlerkurve beschreiben zu können. Zur Verkürzung der Versuchszeit wurden Kettenglieder aus den drei untersuchten Werkstoffen zu einer Kette zusammengesetzt. Die Anzahl der Umläufe bis zum Bruch eines Gliedes wurden erfasst, das defekte Glied wurde ersetzt und die Prüfung solange fortgeführt, bis von jedem Werkstoff fünf Glieder gebrochen waren. Abbildung 5 zeigt die Mittelwerte der aufgenommenen Umlaufzahlen.

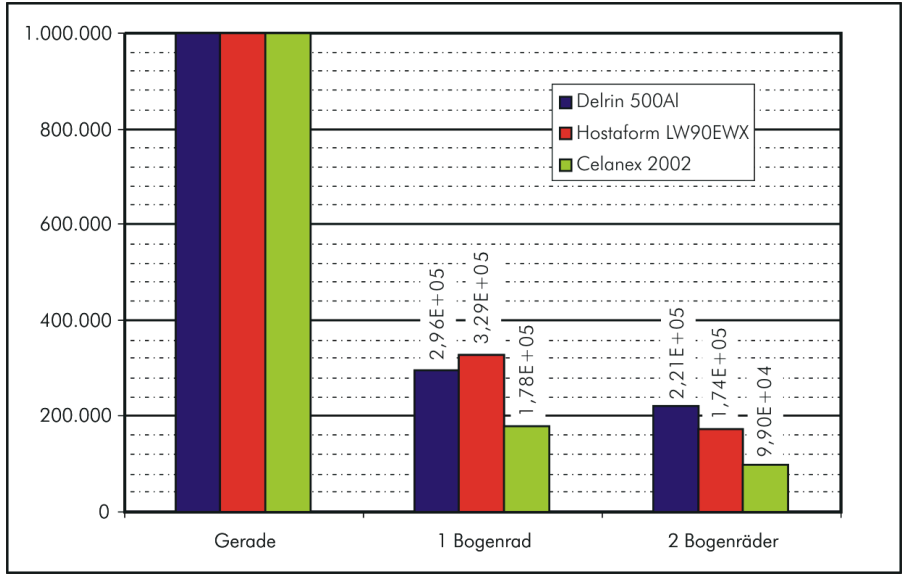

Abb. 5: Mittelwerte der Umlaufzahlen bis zum Bruch

Die Prüfung der geraden Strecke wurde zur Begrenzung der Versuchszeit nach $10^{6}$ Umläufen eingestellt. Da die Ketten in gerader Strecke und Pulser ähnlich belastet werden, ist mit einer angenäherten Übereinstimmung der ertragbaren Schwingspiele zu rechnen. Im Pulser hätten alle Werkstoffe bei $1000 \mathrm{~N}$ mehr als $10^{7}$ Schwingspiele ertragen.

Abbildung 5 macht deutlich, dass bereits bei der Verwendung eines Bogenrades die Anzahl der ohne Bruch ertragenen Umläufe gegenüber der geraden Strecke stark abfällt. Wählt man die $10^{6}$ Lastwechsel der geraden Strecke als Bezugsgröße, werden mit einem Bogenrad nur zwischen 18\% $\left(\right.$ Celanex ${ }^{\circledR}$ ) und 29\% (Delrin ${ }^{\circledR}$ ) davon erreicht. Enthält die Strecke zwei Bogenräder, sinkt die Anzahl der ertragenen Umläufe weiter auf ca. 10 bis $17 \%$ des in der geraden Strecke erreichten Wertes. Dies deckt sich mit der aus der Belastungsanalyse gezogenen Schlussfolgerung, dass Kurven praktisch einen zusätzlichen Lastwechsel mit mehrachsiger Spannung hervorrufen. Je mehr Kurven eine 
Strecke enthält, umso häufiger finden diese überlagerten Lastwechsel bezogen auf einen Umlauf der Kette statt - die ertragbare Anzahl von Umläufen reduziert sich entsprechend. Die Streubreite der Bruchspielzahlen gleicht der bei den Pulserversuchen festgestellten - die Lebensdauer der Ketten aus Celanex ${ }^{\circledR}$ streut mit rund 30\% deutlich stärker als bei den Delrin- und Hostaformketten mit 10\%.

Die großen Reibkräfte und die damit verbundene starke Erwärmung von Gleitschiene und Kette machten es unmöglich, die Bruchspielzahlen der Förderstrecke mit horizontalem Gleitbogen aufzunehmen. Bereits bei einer für Vorversuche gewählten Amplitude von $500 \mathrm{~N}$ schmolz die Kette im Kontaktbereich zum Innenrand der Kurve auf. Um die schnelle Zerstörung der Kette durch den starken Materialabtrag zu verhindern, musste die Prüfung eingestellt werden. Offenkundig limitiert bei der Verwendung von Gleitbögen die thermische Belastbarkeit von Kette und Gleitschiene die ertragbare Belastung und Lebensdauer der Kette.

\subsection{Vergleich der Testmethoden}

Wie bereits erwähnt, erfordert die Festigkeitsprüfung auf Testförderern wesentlich mehr Zeit und Versuchsaufwand, als die Prüfung auf dem Pulser. Anhand einer Gegenüberstellung der Ergebnisse beider Verfahren wurde nach einem Zusammenhang gesucht, der künftig eine Verringerung dieses Aufwandes erlaubt und dennoch praxistaugliche Werte liefert (Abbildung 6).

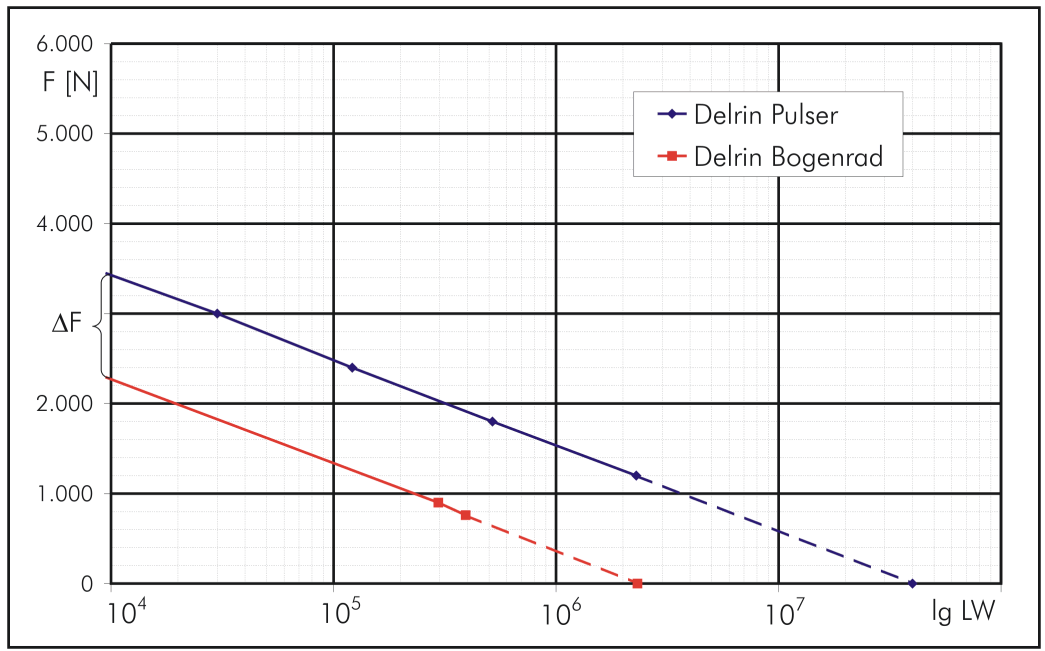

Abb. 6: Vergleich der Wöhlerlinien von Pulser und Testförderstrecke

Die Linien der Testförderer basieren auf den mittleren Bruchspielzahlen bei drei Laststufen. Abbildung 6 zeigt am Beispiel des Werkstoffes Delrin ${ }^{\circledR}$, dass die Kette bei der Prüfung auf der Testförderstrecke zwar insgesamt deutlich geringere Kräfte erträgt, der Festigkeitsabfall in beiden Fällen aber ähnlich ist. Auch bei den anderen beiden Werkstoffen ergab sich dieses Bild. Wenn sich dieser Zusammenhang bei weiteren Kettentypen und Werkstoffen bestätigen lässt, wäre nur noch ein Versuch auf einem Testförderer notwendig, um die Verschiebung $\Delta F$ zu bestimmen. Die Wöhlerlinie der Testförderstrecke ergibt sich dann durch Parallelverschiebung der auf der Prüfmaschine ermittelten Kurve um $\Delta F$.

\section{Abschätzung der Lebensdauer}

\subsection{Vorgeschlagener Ansatz}

Mit der bisher üblichen Dimensionierungsmethode, die sich auf die statische Bruchkraft einer Kette bezog, konnte keine Aussage über die Lebensdauer getroffen werden. Wie die Versuchsergebnisse zeigten, weisen unterschiedliche Werkstoffe einen verschieden starken Festigkeitsabfall auf, der nicht mit der statischen Bruchkraft zusammenhängt. In den Förderstrecken mit Kurven erreichten die Ketten generell nur eine recht geringe Lebensdauer. 
Es wurde nach einem Ansatz gesucht, der eine Abschätzung dieser Lebensdauer und damit eine genauere Dimensionierung erlaubt. Aufgrund der dargestellten, vielfältigen Einflussfaktoren auf Beanspruchung und Lebensdauer waren dazu einige Vereinfachungen erforderlich. Der Zugkraftverlauf beim Durchlaufen einer Förderstrecke wird vereinfacht als schwellende, sinusförmige Last aufgefasst. Im praktischen Einsatz können zusätzlich Zeiten mit wechselnder Belastung des Förderers auftreten. Um diese mathematisch beschreiben zu können, ist eine Zusammenfassung von Zuständen mit ähnlicher Belastung notwendig. Es wird vorgeschlagen, dabei die Betriebszustände Anfahren, Leerlauf, Guttransport, Staubetrieb zu unterscheiden.

Jeder Betriebszustand ist durch eine andere maximale Zugkraft gekennzeichnet. Insgesamt lassen sich die Belastungen als mehrstufiges Lastkollektiv auffassen. Auf dem Gebiet der Betriebsfestigkeit hat die als Palmgren-Miner-Regel bekannte Hypothese einer linearen Schadensakkumulation große Verbreitung erlangt [Zammert85]. Sie erlaubt es, die Lebensdauer eines mehrstufig beanspruchten Bauteils auf im Einstufenversuch ermittelte Wöhlerkurven zurückzuführen. Dazu wird für jede Laststufe die Teilschädigung $D_{i}$ ermittelt, die sich aus dem Quotient von ausgeführten $\left(n_{i}\right)$ und zulässigen Schwingspielen $\left(N_{i}\right)$ ergibt:

$$
D_{i}=\frac{n_{i}}{N_{i}}
$$

Das Bauteil versagt, wenn die Schadenssumme $D$ den Wert eins erreicht.

$$
D=\sum_{i} D_{i}=\sum_{i} \frac{n_{i}}{N_{i}}=1
$$

Bezieht man die Anzahl der Schwingspiele einer Laststufe über deren zeitliche Häufigkeit $H_{i}$ anteilig auf die Gesamtzahl der Schwingspiele $n$ :

$$
n_{i}=H_{i} \cdot n
$$

lässt sich nach Einsetzen dieser Beziehung in Gleichung 2:

$$
D=\sum_{i} \frac{n_{i}}{N_{i}}=\sum_{i} \frac{H_{i} \cdot n}{N_{i}}=n \cdot \sum_{i} \frac{H_{i}}{N_{i}}=1
$$

nach der gesuchten Anzahl ingesamt ertragbarer Schwingspiele umstellen:

$$
n=\frac{1}{\sum_{i} \frac{H_{i}}{N_{i}}}
$$

Diese kann über die gesamte Kettenlänge $L_{G}$ und die Fördergeschwindigkeit $v$ in eine ertragbare Betriebszeit bzw. Lebensdauer $t$ umgerechnet werden:

$$
t=\frac{L_{G}}{v \cdot 3600} \cdot n
$$

Folgendes Beispiel soll verdeutlichen, wie dieser Ansatz auf die Berechnung der Lebensdauer von Kunststoff-Gleitketten angewandt werden kann.

\subsection{Beispielrechnung}

Eine Berechnung nach dem beschriebenen Ansatz erfordert folgende Schritte

1. Berechnung der Zugkraftamplituden,

2. Ermitteln der ertragbaren Umlaufzahlen,

3. Berechnung der Lebensdauer. 
Tab. 1: Gegebene Daten der Betriebsphasen

\begin{tabular}{c|ccc} 
i & Betriebsphase & Häufigkeit & Max. Zugkraft \\
\hline 1 & Leerlauf & 0,2 & $300 \mathrm{~N}$ \\
2 & Transport & 0,5 & $500 \mathrm{~N}$ \\
3 & Staubetrieb & 0,2 & $650 \mathrm{~N}$ \\
4 & Anfahren & 0,1 & $700 \mathrm{~N}$
\end{tabular}

Die Betriebsphasen sind durch ihre Zugkraftamplitude sowie ihre relative Häufigkeit (bezogen auf die gesamte Betriebszeit) gekennzeichnet. Für die Berechnung der Zugkraft wird auf die Gleichungen in den Firmenschriften sowie auf [Auerbach06] verwiesen, die Häufigkeiten sind aus den technologischen Randbedingungen zu ermitteln bzw. einzuschätzen. Für dieses Beispiel sollen die in Tabelle 1 dargestellten Werte angenommen werden.

Nun gilt es, aus dem Festigkeitsschaubild der einzusetzenden Kette die jeder Lastamplitude zugeordnete ertragbare Schwingspielzahl abzulesen. Zur Demonstration der Anwendung des in Abschnitt 2.4 festgestellten Zusammenhanges wird angenommen, dass die Förderstrecke ein Bogenrad enthält. Die ertragbaren Spielzahlen $N_{i}$ der Förderstrecke können aus dem Schaubild für die Prüfmaschine abgelesen werden, wenn zur entsprechenden Zugkraft die Verschiebung $\Delta F$ addiert wird. Es soll eine Delrin-Kette zum Einsatz kommen, für die in den Versuchen ein $\Delta F$ von $1200 \mathrm{~N}$ ermittelt wurde. Abbildung 7 illustriert am Beispiel der Kraftamplitude für den Staubetrieb $\left(F_{3}=650 \mathrm{~N}\right)$ wie der Ablesevorgang erfolgt.

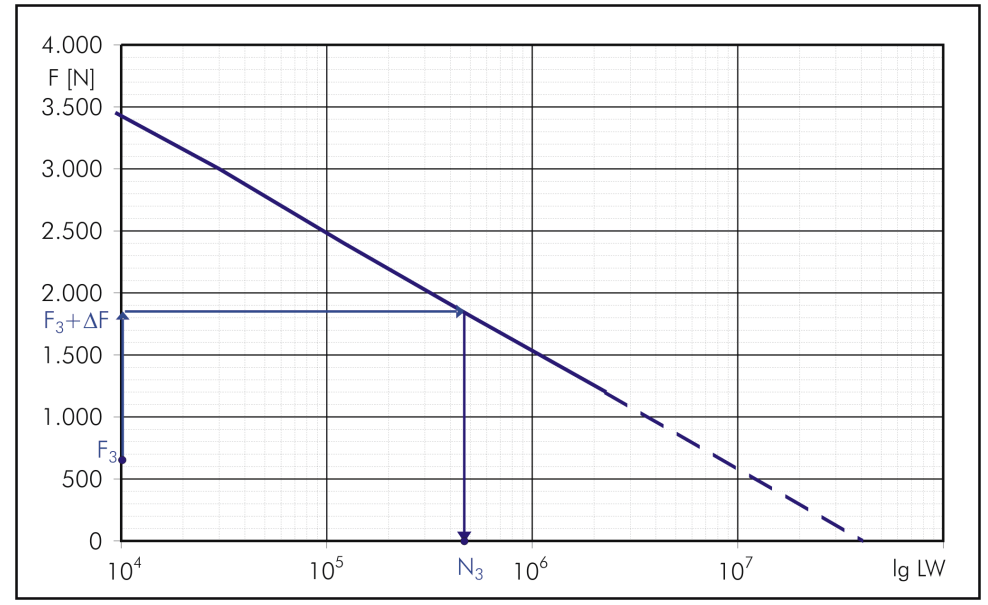

Abb. 7: Ablesen der ertragbaren Umlaufzahl

Hat man dies für alle vier Amplituden durchgeführt, erhält man den in Tabelle 2 dargestellten Datensatz für die Betriebsphasen.

Tab. 2: Kompletter Datensatz der Betriebszustände des Beispiels

\begin{tabular}{c|cccc} 
i & Betriebsphase & Häufigkeit & Max. Zugkraft & Umlaufzahl \\
\hline 1 & Leerlauf & 0,2 & $300 \mathrm{~N}$ & $1,0 \cdot 10^{6}$ \\
2 & Transport & 0,5 & $500 \mathrm{~N}$ & $5,2 \cdot 10^{5}$ \\
3 & Staubetrieb & 0,2 & $650 \mathrm{~N}$ & $4,4 \cdot 10^{5}$ \\
4 & Anfahren & 0,1 & $700 \mathrm{~N}$ & $3,0 \cdot 10^{5}$
\end{tabular}


Mit diesen Daten kann schließlich unter Verwendung von Gleichung 5 die Berechnung der insgesamt ertragbaren Umlaufzahl durchgeführt werden. Nach Einsetzen aller Werte erhält man:

$$
n=\frac{1}{\frac{0,2}{1,0 \cdot 10^{6}}+\frac{0,5}{5,2 \cdot 10^{5}}+\frac{0,2}{4,4 \cdot 10^{5}}+\frac{0,1}{3,0 \cdot 10^{5}}}=5,13 \cdot 10^{5}
$$

Bei einer Kettenlänge von $10 \mathrm{~m}$ und einer Geschwindigkeit von $0,5 \mathrm{~m} / \mathrm{s}$ ergibt sich für die ertragbare Betriebszeit:

$$
t=\frac{10 \mathrm{~m}}{0,5 \mathrm{~m} / \mathrm{s} \cdot 3600 \mathrm{~h} / \mathrm{s}} \cdot 5,13 \cdot 10^{5}=2^{\prime} 850 \mathrm{~h}
$$

Mit dem beschriebenen Ablauf kann die Lebensdauer von Gleitketten bis zu einem Kettenbruch in beliebig gestalteten Förderstrecken näherungsweise berechnet werden. Auf mögliche Fehlerquellen der Berechnung geht folgender Abschnitt ein.

\title{
3.3 Fehlerbetrachtung
}

Der beschriebene Ansatz beruht auf einigen Annahmen und bezieht Größen ein, die teilweise statistisch verteilt sind, so dass die errechnete Lebensdauer mit einer gewissen Unsicherheit behaftet ist. Abbildung 8 zeigt drei Hauptkategorien von Fehlerquellen im Überblick.

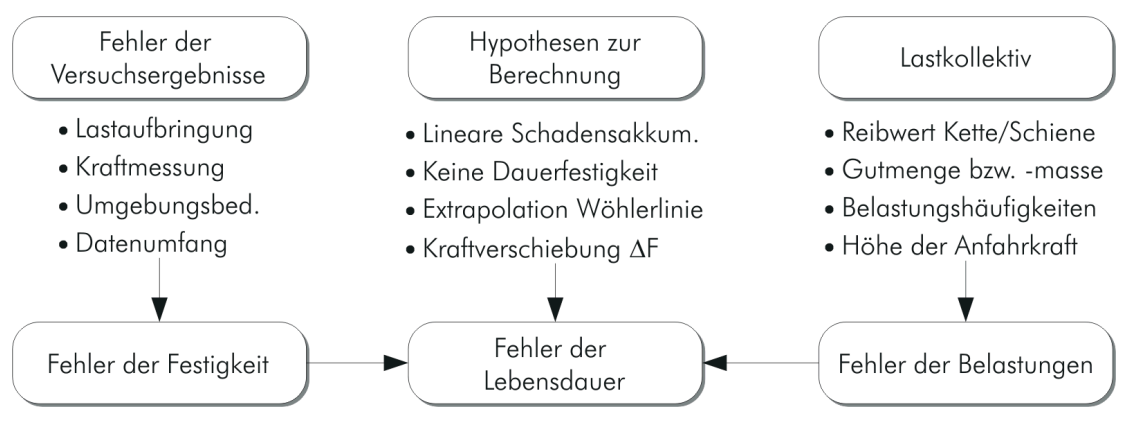

Abb. 8: Gliederung möglicher Fehlerquellen

\begin{abstract}
Zur ersten Kategorie gehören Fehler der experimentell ermittelten Materialkennwerte (Wöhlerkurven), die u.a. durch Toleranzen der Messgeräte und der Lastaufbringung in den Versuchen sowie durch Schwankungen der Materialeigenschaften oder Umweltbedingungen hervorgerufen werden können. Die zweite Gruppe beinhaltet Ungenauigkeiten, die aus den Annahmen bzw. Vereinfachungen des Berechnungsansatzes resultieren. Von der Sorgfalt des Anwenders hängt schließlich der Fehler der letzten Kategorie, des angenommenen Lastkollektivs, ab. Dies betrifft einerseits die Genauigkeit der errechneten Kettenzugkräfte, die vor allem exakte Reibwerte und Gutmengen voraussetzt. Andererseits müssen auch die angenommenen Häufigkeiten der Betriebsphasen möglichst genau dem späteren Einsatzfall entsprechen. Da viele der genannten Fehlerquellen nicht quantifizierbar sind, kann auch der Gesamtfehler der Lebensdauer nicht beziffert werden. In der Literatur dokumentierte Untersuchungen zur Überprüfung der Miner-Regel an Versuchsergebnissen ergaben, dass diese im Mittel zutreffende Ergebnisse liefert [Haibach02]. Allerdings wiesen die ermittelten Schadenssummen je nach Werkstoff, Art und Reihenfolge der Beanspruchungen recht große Streuungen auf [Buxbaum92].

Ungeachtet dessen erlaubt der dargestellte Ansatz erstmals zumindest eine überschlägige Bestimmung der Lebensdauer von Gleitketten aus Kunststoffen. Er stellt damit einen wesentlichen Fortschritt gegenüber der bisherigen Auslegung nach der statischen Bruchkraft und einem pauschalen Sicherheitsfaktor dar, die keinen Aufschluss über die Lebensdauer liefern konnte.
\end{abstract}

\section{Berechnungssoftware}

Um die Dimensionierung von Gleitketten-Fördersystemen nach dem beschriebenen Ansatz zu erleichtern, wurde eine entsprechende Software für PC entwickelt. Dabei wurde besonders auf eine 
benutzerfreundliche Gestaltung der Abläufe und Oberfläche geachtet. Für die Berechnung erforderliche Kennwerte von Antrieben, Ketten und Gleitschienen wurden in einer Datenbank hinterlegt, um die Anzahl der notwendigen Benutzereingaben zu reduzieren. Die übrigen Eingaben erfolgen in vier übersichtlichen Schritten. Abbildung 9 zeigt beispielhaft die Gestaltung des Dialogs zur Festlegung der Förderstrecke, die durch eine schematische Vorschau der Streckenführung unterstützt wird.

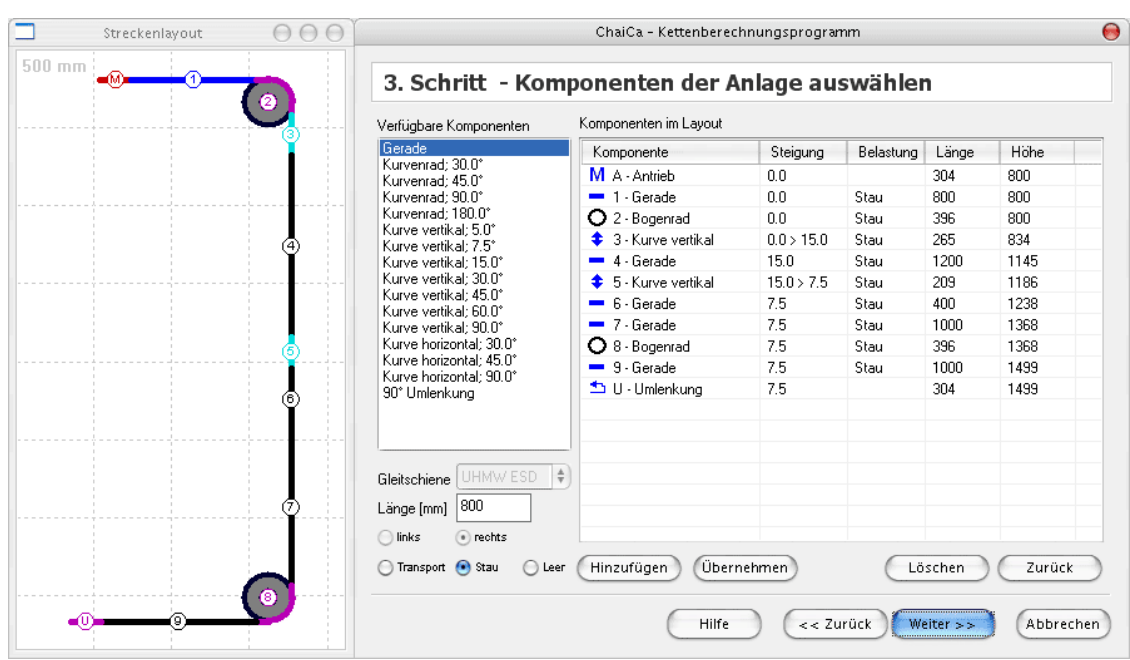

Abb. 9: Dialog zur Festlegung des Streckenverlaufs

Im fünften Schritt werden die Ergebnisse (Zugkraft, Lebensdauer, Antriebsleistung) dargestellt und es kann ein Protokoll der Berechnung gedruckt werden (Abbildung 10).

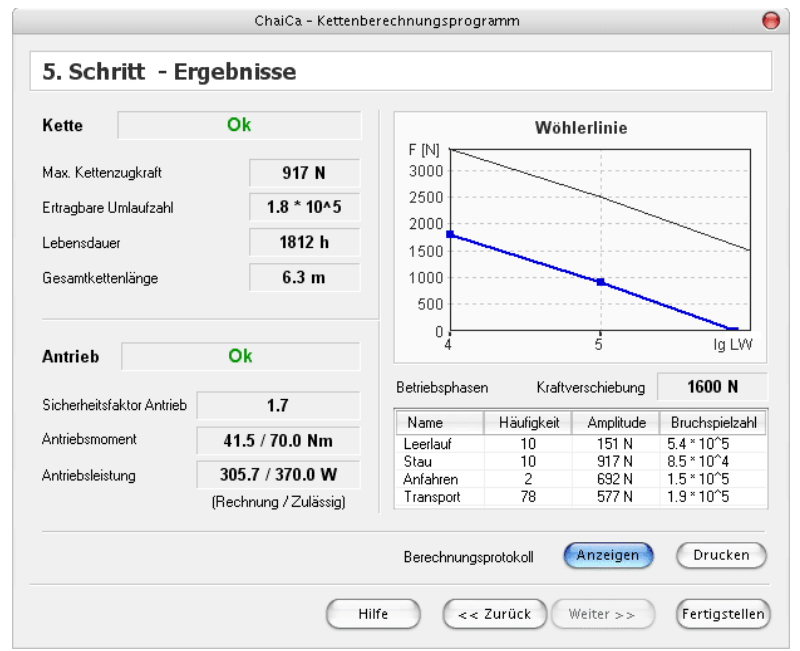

Abb. 10: Anzeige der Ergebnisse

Das Programm existiert derzeit in deutscher und englischer Sprache, Übersetzungen in weitere Sprachen erfordern aufgrund der realisierten Trennung von Beschriftungstexten und Programmcode nur relativ wenig Aufwand. Datenbank und Programm wurden so angelegt, dass mit wenigen Änderungen das Produktsortiment verschiedener Hersteller integriert werden kann. Entsprechend angepasste Programmversionen werden derzeit von mehreren Anbietern von Gleitketten-Fördersystemen getestet.

\section{Zusammenfassung und Ausblick}

Die dargestellten Ergebnisse haben gezeigt, dass die Lebensdauer seitenbeweglicher KunststoffGleitketten nicht nur von der maximalen Gutbelastung bzw. Kettenzugkraft abhängt, sondern auch 
in starkem Maße von der Streckenführung des Förderers. Beim Einsatz von Gleitbögen in einer Förderstrecke bestimmt die thermische Belastbarkeit von Kette und Gleitschienen die Einsatzgrenzen. Die Belastung muss dabei soweit begrenzt werden, dass Aufschmelzen bzw. übermäßiger Verschleiß vermieden wird. Ein Austausch der Kette wird in diesem Fall durch Erreichen einer bestimmten Verschleißgrenze erforderlich, noch bevor ein Ermüdungsbruch auftreten kann.

Werden hingegen Bogenräder verwendet, die zwar die Reibung weitgehend eliminieren, dafür aber die Kettenglieder durch überlagerte Spannungen stärker beanspruchen, begrenzt die Zeitschwingfestigkeit die Lebensdauer der Ketten. Die Minderung der Lebensdauer durch Bogenräder im Vergleich zur ausschließlich geraden Strecke bei gleicher Maximalzugkraft wurde dargestellt. Darüber hinaus wurde ein Ansatz vorgeschlagen, mit dem die Lebensdauer anhand der ermittelten Festigkeitslinien rechnerisch abgeschätzt werden kann. Es wurde darauf hingewiesen, dass viele der einbezogenen Parameter statistisch verteilte Größen darstellen und deshalb unweigerlich zu einer gewissen Unsicherheit bzw. Streuung der errechneten Lebensdauer führen müssen. Dennoch stellt dieser Ansatz einen deutlichen Fortschritt gegenüber der bisherigen Auslegung nach der statischen Bruchkraft dar, weil er zumindest eine überschlägige Beurteilung der zu erwartenden Lebensdauer ermöglicht.

Als Gegenstände weiterer Untersuchungen auf diesem Gebiet empfehlen sich:

- Ermittlung der Dauerfestigkeit weiterer Kettentypen und Werkstoffe, Überprüfung des Zusammenhangs zwischen den Ergebnissen von Prüfmaschine und Testförderstrecke,

- Untersuchung der thermischen Einsatzgrenzen, Schaffung von Dimensionierungsgrundlagen für Reibleistung und Erwärmung,

- Untersuchung von Verschleiß an Platten und Gelenkelementen sowie der Zunahme des Teilungsfehlers, mathematische Beschreibung der Zusammenhänge.

Die Ergebnisse dieser weiterführenden Untersuchungen könnten wertvolle Anregungen zur konstruktiven oder werkstofftechnischen Optimierung der Gleitketten liefern, die eine Steigerung der Belastbarkeit, Lebensdauer und Verschleißfestigkeit der Gleitketten erlauben.

\section{Formelzeichen}

$\begin{array}{ccl}\text { Zeichen } & \text { Einheit } & \text { Beschreibung } \\ D & - & \text { Schadenssumme } \\ D_{i} & - & \text { Teilschädigung der Laststufe i } \\ H_{i} & - & \text { Zeitanteil der Betriebsphase an der Gesamtbetriebszeit } \\ L_{G} & \text { m } & \text { Gesamtlänge der Kette einer Förderstrecke } \\ n & - & \text { Gesamtanzahl der ausgeführten Schwingspiele } \\ n_{i} & - & \text { Ausgeführte Schwingspiele der Laststufe i } \\ N & - & \text { Ertragbare Anzahl von Schwingspielen } \\ t & \mathrm{~h} & \text { Ertragbare Betriebszeit bis zum Bruch } \\ v & \mathrm{~m} / \mathrm{s} & \text { Fördergeschwindigkeit }\end{array}$




\section{Literatur}

[Auerbach06] Untersuchung der Beanspruchung und des Zugkraftverlaufs raumgängiger Gleitketten. In: Logistics Journal (2006)

[Buxbaum92] Buxbaum, Otto: Betriebsfestigkeit: Sichere und wirtschaftliche Bemessung schwingbruchgefährdeter Bauteile. 2. erw. Auflage. Berlin : Verlag Stahleisen, 1992

[Crawford98] Crawford, R.J.: Plastics Engineering. Third Edition. Oxford : ButterworthHeinemann, 1998.

[Haibach02] Haibach, Erwin: Betriebsfestigkeit: Verfahren und Daten zur Bauteilberechnung. 2. Auflage. Berlin, Heidelberg : Springer, 2002.

[Hellerich04] Hellerich, W.; Harsch, G.; Haenle, S.: Werkstoff-Führer Kunststoffe. 9. Auflage. München, Wien : Carl Hanser Verlag, 2004.

[Zammert85] Zammert, W.U.: Betriebsfestigkeitsberechnung. Braunschweig : Vieweg, 1985. 